Original Research Paper

\title{
Binding Mechanism of Bovine Serum Albumin to Troxerutin by Synchronous Fluorescence Spectroscopy and Chemometrics
}

\author{
Tianhu Wang, Yi Yan and Yinsheng Luo \\ School of Electrical and Information Engineering, Jiangsu University of Technology, \\ Changzhou City, Jiangsu Province 213001, P.R. China
}

Article history

Received: 17-02-2016

Revised: 03-05-2016

Accepted: 13-05-2016

Corresponding Author:

Tianhu Wang

School of Electrical and

Information Engineering,

Jiangsu University of

Technology, Changzhou,

Jiangsu Province, China

Email: tianhu2003@126.com

\begin{abstract}
Previous works have reported the binding of Bovine Serum Albumin (BSA) to troxerutin by absorption and fluorescence spectroscopy. In order to extend the investigation of their binding characteristics, the study presented here was designed to evaluate their interaction by the synchronous fluorescence spectroscopy and Multivariate Curve Resolution With Alternating Least Squares (MCR-ALS) under simulative physiological conditions. The Evolving Factor Analysis (EFA) and MCRALS analysis results show that there are three chemical species in the troxerutin-BSA system, which reveal that the fluorescence quenching of BSA caused by troxerutin may be a static quenching mechanism. The spectral profile of each compound was obtained by the soft of MCR-ALS. The obtained average binding constant is $6.43 \times 10^{5} \mathrm{~mol}^{-1} \mathrm{~L}$ for BSA and troxerutin concentration ratio is 7 while that is $6.89 \times 10^{5} \mathrm{~mol}^{-1} \mathrm{~L}$ for troxerutin and BSA concentration ratio is 5 . The study might further help us to better understand the structural features and toxicological action of troxerutin at the molecular level.
\end{abstract}

Keywords: Bovine Serum Albumin (BSA), Troxerutin, Synchronous Fluorescence Spectroscopy, MCR-ALS

\section{Introduction}

Troxerutin is a flavonoid derivative, could be extracted from coffee, tea, cereal grains and a variety of fruits (Marzin et al., 1987). It exerts a great impact on inhibiting the role of red blood cells and platelet aggregation, preventing thrombosis, increasing the content of oxygen in the blood and improving microcirculation etc (Adam et al., 2005). Thank for its pharmaceutical properties, it has been widely used to treat the arteriosclerosis, thrombophlebitis, edema caused by increased vascular permeability, Chronic Venous Insufficiency (CVI) (Pentz et al., 2005). So far, considerable works have been focused on the pharmaceutical properties of troxerutin in order to further to understand its biological activity ( $\mathrm{Lu}$ et al., 2011; Fan et al., 2009; Maurya et al., 2005). Subastri et al. (2015) reported its interaction with transfer RNA and revealed that it may have potential therapeutic application. Heidarzadeh et al. (2014) showed that troxerutin may reduce tissue injury and lipid peroxidation. Wang et al. (2009) studied the binding of troxerutin to Bovine Serum Albumin (BSA) and revealed that the fluorescence quenching of BSA caused by troxerutin is a static quenching procedure.

Serum albumins are the most abundant protein constituents of blood plasma, which are responsible for maintaining the $\mathrm{pH}$ in blood and transporting drugs in living organisms (Anbazhagan and Renganathan, 2008; Kragh-Hansen, 1981). Among the serum albumins, BSA has been widely used in biophysical studies to clarifying the binding mechanism of small molecules to proteins due to its physiological functions and $76 \%$ of structural similarity with Human Serum Albumin (HSA) (Sułkowska, 2002; Dasmandal et al., 2015). Investigation on binding of drugs to serum albumins may help us to understand drug's distribution and toxicity in the biological systems.

In recent years, several optical techniques have been carried out to clarify the interaction mechanism of drugs with serum albumins, such as UV-vis and fluorescence 
spectroscopy, Raman spectroscopy and Circular Dichroism (CD) and so on. Among these methods, the spectrophotometry is the most common and convincing methods (Cui et al., 2004; Lin and Koenig, 1976; Gelamo et al., 2002). With the development of chemometrics algorithms and high precision instruments, multi-dimensional data of complex reaction system could be easily obtained. MCR-ALS is a very powerful tool to handle deviations from trilinearity because it is easy to deal with the data and also, it is a powerful chemometrics tool (Ni et al., 2011; Azzouz and Tauler, 2008).

In the previous study, we have reported the binding mechanism of troxerutin to BSA by spectral method involving fluorescence spectroscopy (Wang et al., 2009). The binding distance, binding site, binding consants and the effect of troxerutin on the conformation of BSA were studied. In order to extend to the investigation of their interactions, the study presented here was designed by using the synchronous fluorescence spectroscopy and MCRALS under simulative physiological conditions. The number of chemical species that present in the interaction system of troxerutin with BSA was analyzed by using Evolving Factor Analysis (EFA) based on synchronous fluorescence spectroscopy data. Subsequently, the spectral profile of each compound was achieved by a soft of MCR-ALS.

\section{Materials and Methods}

\section{Materials and Apparatus}

Troxerutin was procured from Shanxi Yabao of China. The troxerutin solution $\left(3.0 \times 10^{-3} \mathrm{~mol} \mathrm{~L}^{-1}\right)$ was prepared in phosphate buffer solution of $\mathrm{pH}$ 7.4. BSA was obtained from Boquan Biochemical Technology of China and has been used without further purification. The stock solution of BSA $\left(3.0 \times 10^{-5} \mathrm{~mol} \mathrm{~L}{ }^{-1}\right)$ was prepared in phosphate buffer solution of $\mathrm{pH} 7.4$ which containing $0.1 \mathrm{~mol} \mathrm{~L}^{-1} \mathrm{NaCl}$. The synchronous spectra were recorded by spectrofluorophotometer of RF5301PC (Shimadzu, Japan) equipped with a quartz cell of $1.0 \mathrm{~cm}$ at room temperature. The slit widths of emission and excitation were used in $5.0 \mathrm{~nm}$.

\section{Experimental Procedure}

Experiment 1: The BSA concentration was kept at $1.0 \times 10^{-6} \mathrm{~mol} \mathrm{~L}^{-1}$, then, different concentration range $\left(0.0-7.5 \times 10^{-6} \mathrm{~mol} \mathrm{~L}^{-1}\right.$ at intervals $0.5 \times 10^{-6} \mathrm{~mol} \mathrm{~L}^{-1}$, total 16 solutions) of troxerutin was added.

Experiment 2: The troxerutin concentration was kept at $1.0 \times 10^{-6} \mathrm{~mol} \mathrm{~L}^{-1}$, then, different concentration range $\left(0.0-7.5 \times 10^{-6} \mathrm{~mol} \mathrm{~L}^{-1}\right.$ at intervals $0.5 \times 10^{-6} \mathrm{~mol} \mathrm{~L}^{-1}$, total 16 solutions) of BSA was added.

\section{Evolving Factor Analysis (EFA)}

The Evolving Factor Analysis (EFA) is a chemometric technique of identifying the nature structure of multivariate observation and treating dimension reduction, has been used to analyze the number of chemical species (Amrhein et al., 1996; Maeder, 1987). EFA is calculated from two directions, namely forward direction and reverse direction. The eigenvalue of the $\boldsymbol{X}_{\mathrm{i}}$ sub array can be calculated by the following formula:

$X_{\mathrm{i}}=Y U^{\mathrm{T}}$

where, $Y(I \times N)$ is the score matrix, $U^{\mathrm{T}}(N \times N)$ is the loading matrix, $\mathrm{N}$ is the number of pure substance, $X_{\mathrm{i}}$ is composed of the $i=1,2, \ldots$ line of the data matrix $X$.

The main idea of EFA is to follow the evolution or change of the rank of $Y$ with progressing elution by rank analysis of the sub matrices $Y$. The appearance of each new component at the detector is intrinsically associated with the increase of the rank by one. Obviously there has to be a minimal difference in the retention times and, further, the spectra data have to be linearly independent.

\section{Multivariate Curve Resolution-Alternating Least Squares (MCR-ALS)}

The soft of MCR-ALS, a recently developed method, could evaluate the concentration and spectral profile of the chemical species for multivariate data (Maeder and Zilian, 1988).

In order to obtain the concentration profile and pure spectra of spectroscopic ally active components in the drug-protein system, the soft of MCR-ALS procedure was adopted to analyze the experimental data matrix $D$ $\left(N_{\mathrm{C}} \times N_{\mathrm{S}}\right)$. The $D$ can be decomposed according to the following equation (Jaumot et al., 2005):

$D=C S^{T}+E$

where, $C$ and $S^{T}$ is the concentration profiles with the dimension of $N_{\mathrm{C}} \times N$ and spectral profiles with the dimension of $N \times N_{\mathrm{S}}$; respectively. $E\left(N_{\mathrm{C}} \times N_{\mathrm{S}}\right)$ is the residual matrix containing the data variance unexplained by $C S^{\mathrm{T}} . N_{\mathrm{C}}$ is the number of spectra recorded at different concentration ratio of ([BSA]: [Troxerutin], $N$ is the number of the detected chemical species and $N_{\mathrm{S}}$ is the number of the wavelengths.

In order to eliminate the uncertainties of rotation and solve the problem of low matrix resolution, an extended matrix was used in this study. For this purpose, two kinds of experiments were designed to obtain the data matrix $D^{\mathrm{BSA}}$ and $D^{\text {Troxerutin }}$ (see experiment 1 ). The extended matrix can be obtained from the synchronous spectroscopy data matrix at two modes of experiment 1 and 2, which are overlapped in the column direction. The 
rows of new matrix are the sum of two rows of a single matrix. The extended matrix can be expressed as Equation 3:

$\left[\begin{array}{l}D^{B S A} \\ D^{\text {Troxerutin }}\end{array}\right]=\left[\begin{array}{l}C^{B S A} \\ C^{\text {Troxerutin }}\end{array}\right] S^{T}+\left[\begin{array}{l}E^{B S A} \\ E^{\text {Troxerutin }}\end{array}\right]$

where, $\left[D^{\mathrm{BSA}} ; D^{\text {Troxerutin }}\right]$ is the column-wise augmented matrix. The $D^{\text {Troxerutin }}$ and $D^{\mathrm{BSA}}$, correspond to two different experiments, 1 and 2, respectively (see experiment 2 ).

\section{Results and Discussion}

\section{The Synchronous Fluorescence Spectroscopy of Troxerutin -BSA Interaction}

Figure 1 and 2 show the synchronous fluorescence spectroscopy of the binding of troxerutin to BSA in the two different experiments 1 and 2 , in which $\Delta \lambda$ is equal to $20 \mathrm{~nm}$, under simulative physiological conditions. According to Fig. 1, the synchronous fluorescence intensity of BSA gradually decreased with the increasing the troxerutin concentration, which may indicate that the binding of troxerutin to BSA had occurred as reported in earlier work. Further, the maximum emission wavelength of BSA show a slight blue shift, which indicates that the micro-environment around of BSA has been changed (Singh et al., 2015; Tao et al., 2015). From Fig. 2, one can see that the synchronous fluorescence intensity of troxerutin gradually increased with increasing the BSA concentration.

However, it is very difficult to confirm whether the reaction has occurred between troxerutin and BSA and cannot directly determine that the quenching of spectroscopy is fluorescence quenching or spectral superposition. In order to further study the binding of troxertuin to BSA, the synchronous fluorescence spectroscopy data was studied by combination of EFA and MCR-ALS methods.

\section{The Chemical Species Analysis}

Figure 3 shows the EFA plots of the synchronous spectroscopy between troxerutin and BSA. The blue horizontal line was retrieved by related noise and EFA. According to (Amrhein et al., 1996), the number of significant factors containing the spectral evolution recorded degradation process, which is equal to the number of chemical species contributing to the analyzed spectra or the number of independent reactions +1 . In the forward analysis, the first degradation variability of spectral data can be explained by two factors. In the beginning of the reaction, the presence of two factors must be corresponding to two chemical species of the process, in which BSA is degraded. The ANOVA result show that there is an obvious significant both of forward and backward analysis. According to Fig. 3, a new factor becomes relevant after a certain time, which indicates that another variability source has appeared, that is Troxerutin-BSA complex. At last, no additional factor is obtained. In the backward, similar information could be obtained from Fig. 3. According to the discussion above, it is reasonable to propose that at least three chemical species active (BSA, troxerutin and Troxerutin-BSA complex) involved in the process. Moreover, it is further confirmed that the fluorescence quenching of BSA caused by troxerutin through a static quenching procedure. The behavior of each chemical species can be obtained by the soft of MCR-ALS (Fig. 4 and 5). These results are good agreement with the previous work, which reported the binding of troxerutin to BSA by absorption and fluorescence spectroscopy. Contrary to previous work, this paper presents the methods combination of synchronous fluorescence spectroscopy and MCR-ALS to study the interaction of troxerutin with BSA and provide more information for understanding their binding characteristics.

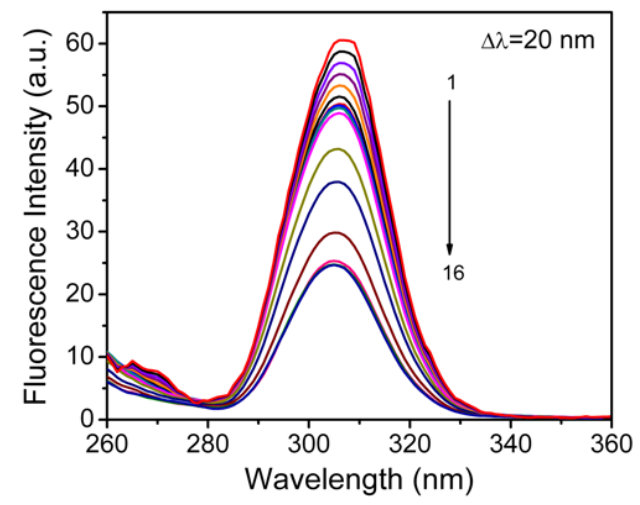

Fig. 1. The synchronous fluorescence spectroscopy of the binding of BSA to troxerutin (the curves from 1 to 16 [Troxerutin]: $[\mathrm{BSA}]=0,0.5,1.0, \ldots, 7.5)$

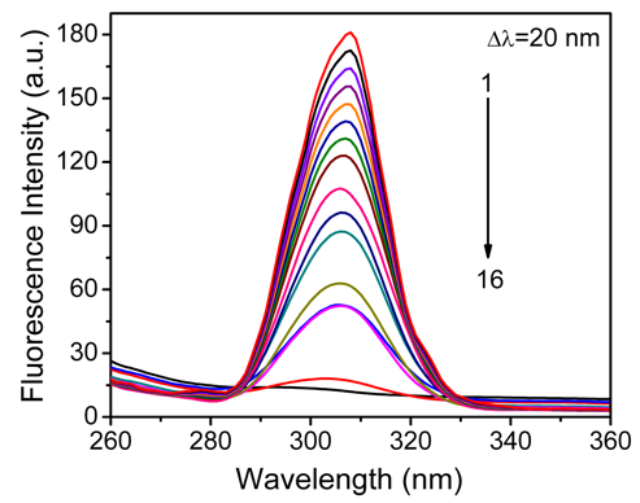

Fig. 2. The synchronous fluorescence spectroscopy of the binding of BSA to troxerutin (the curves from 1 to 16 $[\mathrm{BSA}]:[$ Troxerutin $]=7.5,6.0,5.5, \ldots 0)$ 


\section{The MCR-ALS Analysis}

As the synchronous fluorescence spectra overlap each other (see from Fig. 1 and 2), it is very difficult to determine the possibility of the interaction between troxerutin and BSA. In order to solve this problem, the extended matrix of the synchronous fluorescence spectra data was set up and was analyzed by soft MCR-ALS. Moreover, by using this method, we can obtain the pure substance spectra of the various components based on the MCR-ALS analysis. These results help us to understand the binding mechanism of troxerutin to BSA.

Figure 4 and 5 show the pure substance spectroscopy and the curve of concentration trend, respectively. According Fig. 4 and 5, it can be concluded that there are three chemical species in the Troxerutin-BSA system, which is fully consistent with the results of EFA analysis. The results confirm that troxerutin can interact with BSA from a Troxerutin-BSA complex, thus, it can

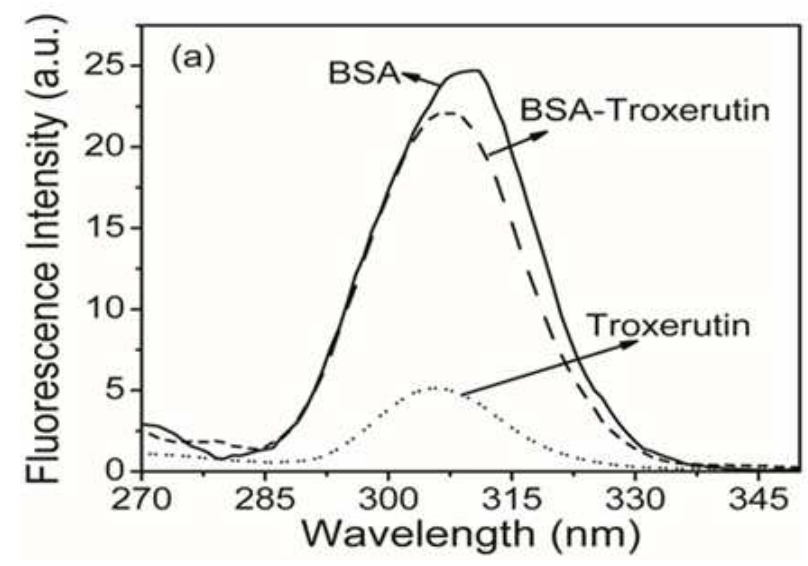

be concluded that the quenching mechanism of BSA caused by troxerutin is a static quenching.

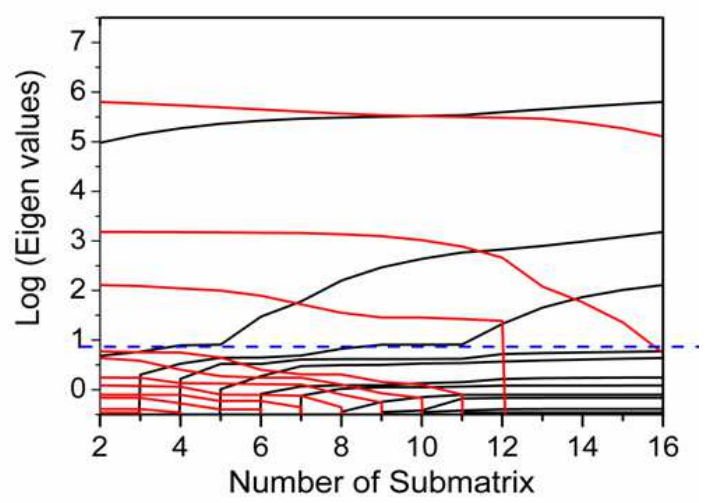

Fig. 3. The EFA plots of the synchronous fluorescence spectroscopy of the binding of BSA to troxerutin. Forward (black lines) and backward (red lines) directions

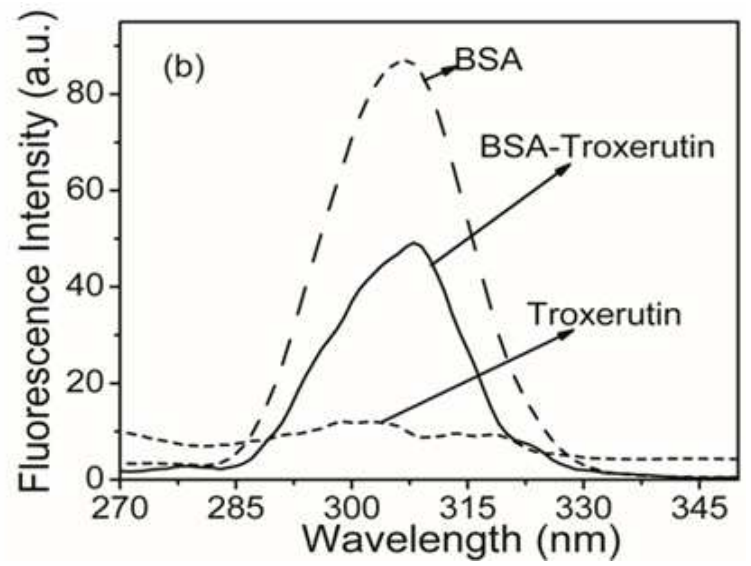

Fig. 4. The pure substance spectroscopy of MCR-ALS analysis (a) experiment 1; (b) experiment 2
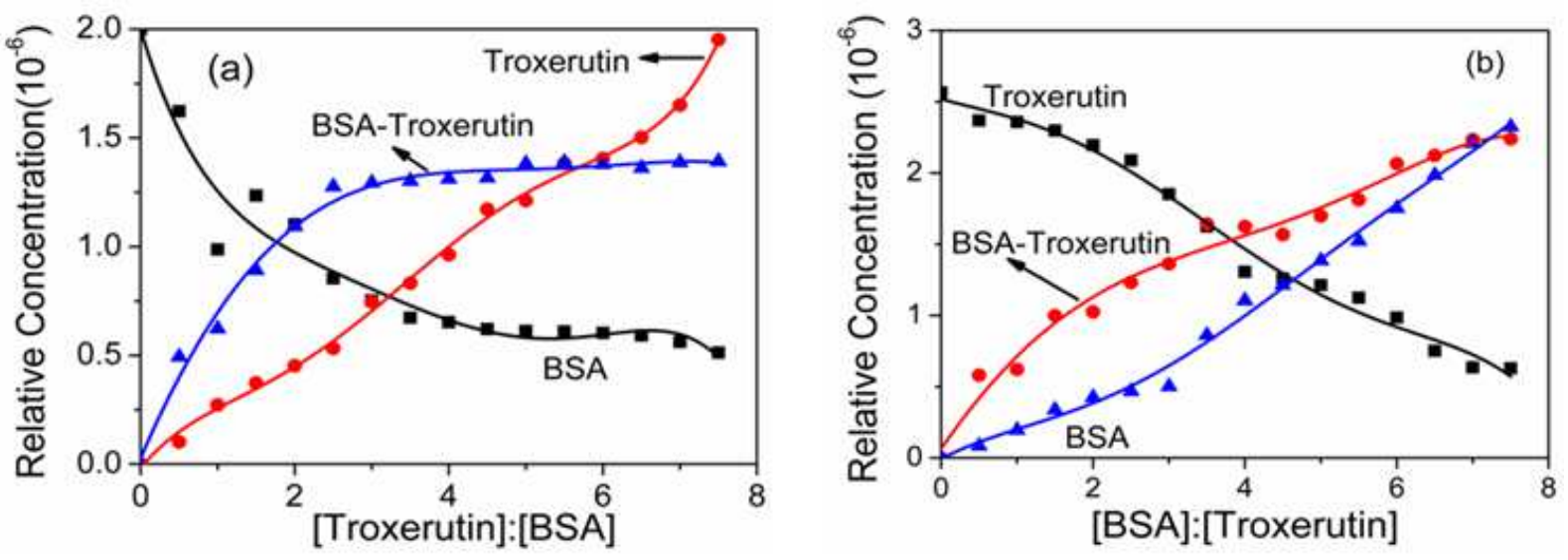

Fig. 5. The concentration trend of MCR-ALS analysis of the interaction between BSA with troxerutin (a) experiment 1; (b) experiment 2 
According to Fig. 5a, it can be seen that the concentration of BSA gradually decrease and the complex of Troxerutin-BSA increase with the increasing concentration of troxerutin. From Fig. 5b, the concentration of Troxerutin-BSA complex gradually increase and the degradation of free troxerutin with the added BSA.

Moreover, the concentrations of Troxerutin-BSA complex remained stable as the [BSA]: [Troxerutin] or [Troxerutin]: [BSA] mole ratio in the complex is about 7 and 5, respectively, showing that one troxerutin molecule was bound to one base pair (Anbazhagan and Renganathan, 2008). The average value for the binding constant was obtained from Equation 4:

$$
K_{a p p}=[\text { BSA }- \text { Troxerutin }] /[\mathrm{BSA}][\text { Troxerutin }]
$$

where, [BSA-Troxerutin], [BSA] and [Troxerutin] is the concentration of free troxerutin-BSA complex, BSA and troxerutin respectively.

According to Equation 4, the average value of the binding constants were calculated as $6.43 \times 10^{5}$ at the [BSA]:[Troxerutin] $=7$ and $6.89 \times 10^{5} \mathrm{~mol}^{-1} \mathrm{~L}$ at the $[$ Troxerutin]:[BSA] $=5$. The prediction of two kinds of dropping models is good agreement with experimental results drawn from MCR-ALS, which indicate that the spectra and concentration profiles of Troxerutin-BSA complex were correctly clarified.

\section{Conclusion}

The binding of troxerutin to BSA was studied by combination of the synchronous fluorescence spectroscopy and MCR-ALS method. The EFA and MCR-ALS analysis results showed that there are three chemical species in the Troxerutin-BSA system, detailed analysis revealed that the fluorescence quenching of BSA caused was a static quenching mechanism. Meanwhile, the average binding constant was obtained as $6.43 \times 10^{5} \mathrm{~mol}^{-1} \mathrm{~L}$ at the [BSA]:[Troxerutin] $=7$ and $6.89 \times 10^{5} \mathrm{~mol}^{-1} \mathrm{~L}$ at the [Troxerutin]:[BSA] $=5$.

\section{Acknowledgement}

The authors would like to thank the funding support from the Natural Science Foundation of Jiangsu Province (Grants No. BK20150247) and Ministry of housing and Urban-Rural Development of China (No. 2015-R1-021).

\section{Author's Contributions}

Tianhu Wang: Planning and performing the experiments and data interpretation.

Yi Yan: Data test.

Yinsheng Luo: Data analysis.

\section{Ethics}

This article is original and contains unpublished material. The corresponding author confirms that all the other authors have read and approved the manuscript and no ethical issues involved.

\section{References}

Adam, B.S., R. Pentz, C.P. Siegers, O. Strubelt and M. Tegtmeierb, 2005. Troxerutin protects the isolated perfused rat liver from a possible lipid peroxidation by coumarin. Phytomedicine, 12: 52-61. DOI: 10.1016/j.phymed.2004.01.007

Amrhein, M., B. Srinivasan, D. Bonvin and M.M. Schumacher, 1996. On the rank deficiency and rank augmentation of the spectral measurement matrix. Chemom. Intell. Lab. Syst., 33: 17-33. DOI: 10.1016/0169-7439(95)00086-0

Anbazhagan, V. and R. Renganathan, 2008. Study on the binding of 2,3-diazabicyclo[2.2.2]oct-2-ene with bovine serum albumin by fluorescence spectroscopy. J. Lumin., 128: 1454-1458.

DOI: $10.1016 /$ j.jlumin.2008.02.004

Azzouz, T. and R. Tauler, 2008. Application of Multivariate Curve Resolution Alternating Least Squares (MCR-ALS) to the quantitative analysis of pharmaceutical and agricultural samples. J. Am. Podiatric Med. Assoc., 74: 1201-10. DOI: 10.1016/j.talanta.2007.08.024

Cui, F.L., J. Fan, J.P. Li and Z.D. Hu, 2004. Interactions between 1-benzoyl-4-p-chlorophenyl thiosemicarbazide and serum albumin: investigation by fluorescence spectroscopy. Bioorg. Med. Chem., 12: 151-157. DOI: 10.1016/j.bmc.2003.10.018

Dasmandal, S., A. Kundu, S. Rudra and A. Mahapatra, 2015. Binding interaction of an anionic amino acid surfactant with bovine serum albumin: Physicochemical and spectroscopic investigations combined with molecular docking study. RSC Adv., 58: 79107-79118. DOI: 10.1039/C5RA17254C

Fan, S.H., Z.F. Zhang, Y.L. Zheng, J. Lu and D.M. Wu et al., 2009. Troxerutin protects the mouse kidney from d-galactose-caused injury through anti-inflammation and anti-oxidation. Int. Immunopharmacol., 9: 91-96. DOI: $10.1016 /$ j.intimp.2008.10.008

Gelamo, E.L., C.H.T.P. Silva, H. Imasato and M. Tabak, 2002. Interaction of Bovine (BSA) and Human (HSA) serum albumins with ionic surfactants: Spectroscopy and modelling. Biochim. Biophys. Acta, 1594: 84-99. DOI: $10.1016 / \mathrm{S} 0167-4838(01) 00287-4$ 
Heidarzadeh, F., R. Badalzadeh and H. Hatami, 2014. The effect of troxerutin on lipid peroxidation and tissue injury induced by myocardial ischemia reperfusion injury in diabetic rat. Razi J. Med. Sci., 12: 37-45.

Jaumot, J., R. Gargallo, A. de Juan and R. Tauler, 2005. A graphical user-friendly interface for MCR-ALS: A new tool for multivariate curve resolution in MATLAB. Chemometr. Intell. Lab. Syst., 76: 101-110. DOI: 10.1016/j.chemolab.2004.12.007

Kragh-Hansen, U., 1981. Molecular aspects of ligand binding to serum albumin. Pharmacol. Rev., 33: 17-53. PMID: 7027277

Lin, V.J.C. and J.L. Koenig, 1976. Raman studies of bovine serum albumin. Biopolymers, 15: 203-218. DOI: 10.1002/bip.1976.360150114

Lu, J., D.N. Wu, Z.H. Zheng, Y.L. Zheng and B. Hu et al., 2011. Troxerutin protects against high cholesterolinduced cognitive deficits in mice. Brain, 134: 783-797. DOI: 10.1093/brain/awq376

Maeder, M. and A. Zilian, 1988. Evolving factor analysis, a new multivariate technique in chromatography. Chemometr. Intell. Lab. Syst., 3: 205-213. DOI: 10.1016/0169-7439(88)80051-0

Maeder, M., 1987. Evolving factor analysis for the resolution of overlapping chromatographic peaks. Anal. Chim. Acta, 181: 527-530. DOI: $10.1021 / \mathrm{ac} 00130 \mathrm{a} 035$

Marzin, D., H.V. Phi, P. Olivier and J. Sauzieres, 1987. Study of mutagenic activity of troxerutin, a flavonoid derivative. Toxicol. Lett., 35: 297-305. DOI: $10.1016 / 0378-4274(87) 90219-0$

Maurya, D.K., S. Balakrishnan, V.P. Salv and C.K.K. Nair, 2005. Protection of cellular dna from $\gamma$-radiation-induced damages and enhancement in DNA repair by troxerutin. Molecular Cellular Biochem., 280: 57-68.

DO: $10.1007 / \mathrm{s} 11010-005-8052-3$
Ni, Y., Y. Wang and S. Kokot, 2011. Study of the interaction between 10-hydroxycamptothecine and DNA with the use of ethidium bromide dye as a fluorescence probe. Sensor. Actuat. B, 156: 290-297. DOI: $10.1016 /$ j.snb.2011.04.035

Pentz, R., C.P. Siegers, O. Strubelt and M. Tegtmeier, 2005. Troxerutin protects the isolated perfused rat liver from a possible lipid peroxidation by coumarin. Phytomedicine, 12: 52-61. PMID: 15693708

Singh, S., K. Sharma and S.K. Awasthi, 2015. The interaction of (7-chloroquinolin-4-yl)-(2,5dimethoxyphenyl)-amine hydrochloride dihydrate with serum albumin proteins, inputs from spectroscopic, molecular docking and X-ray diffraction studies. RSC Adv., 5: 85854-85861. DOI: $10.1039 / \mathrm{c} 5 \mathrm{ra} 02815 \mathrm{a}$

Subastri, A., C.H. Ramamurthy, A. Suyavaran and P. Lokeswara Raoa et al., 2015. Probing the interaction of troxerutin with transfer RNA by spectroscopic and molecular modeling. J. Photoch. Photobio. B, 153: 137-144. DOI: $10.1016 /$ j.jphotobiol.2015.09.013

Sułkowska, A., 2002. Interaction of drugs with bovine and human serum albumin. J. Molecular Struct., 614: 227-232. DOI: 10.1016/S0022-2860(02)00256-9

Tao, M., G. Zhang, C. Xiong and J. Pan, 2015. Characterization of the interaction between resmethrin and calf thymus DNA in vitro. New J. Chem., 39: 3665-3674. DOI: 10.1039/C4NJ02321H

Wang, T.H., Z.M. Zhao, L. Zhang and L. Ji, 2009. Spectroscopic studies on the interaction between troxerutin and bovine serum albumin. J. Mol. Struct., 937: 65-69. DOI: 10.1016/j.molstruc.2009.08.015 